\title{
Histopathological findings in oral lichen planus and their correlation with the clinical manifestations
}

\author{
Francisca Fernández-González ${ }^{1}$, Rocío Vázquez-Álvarez ${ }^{2}$, Dolores Reboiras-López ${ }^{3}$, Pilar Gándara-Vila ${ }^{4}$, \\ Abel García-García ${ }^{5}$, José-Manuel Gándara-Rey ${ }^{6}$
}

\footnotetext{
${ }^{1}$ Dentist and Student of the Master's in Oral Medicine, Oral Surgery and Implantology School of Medicine and Dentistry of the University of Santiago de Compostela

${ }^{2}$ Dentist and Student of the Master's in Oral Medicine, Oral Surgery and Implantology School of Medicine and Dentistry of the University of Santiago de Compostela

${ }^{3}$ Dentist with a Master's in Oral Medicine, Oral Surgery and Implantology

${ }^{4}$ Dentist with a Master's in Oral Medicine, Oral Surgery and Implantology

${ }^{5}$ Maxillofacial Surgeon, Professor of Oral Surgery and Director of the Master's in Oral Medicine, Oral Surgery and Implantology at the School of Medicine and Dentistry of the University of Santiago de Compostela. Head of the Department of Maxillofacial Surgery at the Provincial Hospital of Santiago de Compostela

${ }^{6}$ Stomatologist, Full Professor of Oral Medicine and Director of the Master's in Oral Medicine, Oral Surgery and Implantology at the School of Medicine and Dentistry of the University of Santiago de Compostela
}

Correspondence:

School of Dentistry

c/ Entrerrios, $s / n$

15782 Santiago de Compostela-

A Coruña (Spain)

josemanuel.gandara@usc.es

Received: 07/01/2010

Accepted: 18/03/2010
Fernández-González F, Vázquez-Álvarez R, Reboiras-López D, GándaraVila P, García-García A, Gándara-Rey JM. Histopathological findings in oral lichen planus and their correlation with the clinical manifestations. Med Oral Patol Oral Cir Bucal. 2011 Aug 1;16 (5):e641-6. http://www.medicinaoral.com/medoralfree01/v16i5/medoralv16i5p641.pdf

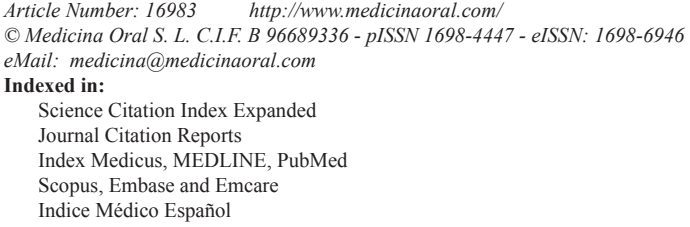

\begin{abstract}
Objectives: To highlight the most characteristic histopathological findings of oral lichen planus and their correlation with the clinical manifestations and forms.

Study design: We performed a retrospective study of 50 biopsied and diagnosed cases of oral lichen planus obtained over a period of 11 years, spanning from May 1998 to April 2009. We analyzed the age and sex of the patient, type of lichen planus, location and different histopathological findings, comparing them with the clinical lesions.

Results: Seventy eight percent of the patients are female and 22\% are male, with an average age of 56.06 years for both sexes. The most frequent clinical form is reticular, present in $78 \%$ of the cases, and the most common location is the buccal mucosa, present in $70 \%$ of the patients. Hydropic degeneration of the basal layer and lymphocytic infiltration in the subepithelial layer are observed in the entire sample. Signs of atypia were identified in $4 \%$ of the cases, but without dysplasic features. Other common histological findings were the presence of necrotic keratinocytes (92\%), hyperplasia (54\%), hyperkeratosis (66\%), acanthosis (48\%), and less frequently, serrated ridges (30\%) and the presence plasma cells $(26 \%)$.
\end{abstract}


Conclusions: Oral lichen planus is a disease that is more common in women, usually appearing in the fifth and sixth decades of life. The most common clinical form is reticular, manifesting mainly in the buccal mucosa. Histological findings characteristic of oral lichen planus include hydropic degeneration of the basal layer, lymphocytic infiltration in the subepithelial layer and the absence of epithelial dysplasia; however, it is also frequent to observe hyperplasia phenomena at the epithelial level, hyperkeratosis, acanthosis and the presence of necrotic keratinocytes.

Key words: Oral lichen planus, histopathological findings, clinical manifestations.

\section{Introduction}

Oral lichen planus is a chronic inflammatory and autoimmune disease (1), affecting approximately 1 to $2 \%$ of the population (2), mainly females, and manifesting most frequently during the fifth and sixth decades of life (3). It is currently considered a disease of unknown etiology and with a multifactorial pathogenesis (1). It is believed that the T CD8+ lymphocytes-which are responsible for apoptosis that occurs at the epithelial level-play a fundamental role in its manifestation (4). Oral lichen planus can manifest in the oral mucosa, skin, nails, scalp and other mucosas. In general, oral lesions usually appear before skin lesions, and sometimes the lesions only appear orally (5). In the mouth, the area most commonly affected is the buccal mucosa, although it may also manifest on the tongue, gingiva and/or the palate (3).

Various clinical classifications of oral lichen planus have been proposed. These classifications include that suggested by Silverman, who distinguishes three types: reticular, atrophic and erosive (6). The reticular form usually appears symmetrically on the buccal mucosa and presents few symptoms (6). It is common to observe the atrophic form at the buccal, lingual and/or gingival level, with the latter appearing in the form of desquamative gingivitis (1). The erosive form primarily manifests in the buccal mucosa and lingual dorsum, and it along with the atrophic form, presents the most symptoms (7).

The diagnosis of oral lichen planus is first obtained based on the clinical appearance of the lesions, and is subsequently confirmed by a biopsy and a histopathological study (7). The majority of authors agree that a biopsy is necessary, given that it allows us to confirm the clinical diagnosis and make the differential diagnosis with other lesions (7).

There are a number of lesions that are similar to oral lichen planus, both from a clinical as well as a histological standpoint. They are called "lichenoid reactions", which have a known cause and include lichenoid contact lesions, drug-induced lichenoid reactions and lichenoid reactions in graft versus host reaction (8). The difference between a lichen planus and a lichenoid reaction is determined by a series of clinical and histological criteria of the lichen planus itself, which the lichenoid reaction does not meet in its entirety (9).
The clinical criteria include the bilateral presence of symmetrical lesions and white reticular lesions. The lesions may be atrophic, erosive, bullous or manifest in the form of plaque, appearing along with reticular lesions in a given area of the oral cavity. If both criteria are met, it is considered a typical lichen planus. Lesions that simulate a lichen planus but do not meet the above criteria are considered to be clinically compatible with lichen planus.

The histological criteria include the existence of a band of lymphocytic inflammatory infiltrate in the subepithelial connective tissue, hydropic degeneration of the basal layer and the absence of epithelial dysplasia. If the above three criteria are met, the lesion is considered a typical lichen planus from a histological perspective; and as for those that do not meet one of the histological criteria, they are considered to be lesions that are histologically compatible with lichen planus.

The differential diagnosis between lichen planus and lichenoid reaction will be based on the combination of the clinical and histological aspects previously mentioned (9). Thus, all of the clinical and histological criteria must be met in the case of lichen planus. Conversely, lichenoid reaction includes patients with typical lichen planus clinically but not histologically, patients with typical lichen planus histologically but not clinically, and patients who are both clinically and histologically only compatible with lichen planus (9).

The objective of treating lichen planus is to control the different outbreaks that exist, given that the lesions are usually not completely cured. Such treatment differs according to the clinical form present in each case. Generally, the reticular forms are not treated, whereas the atrophic and erosive forms are primarily treated with topical corticosteroids. In cases where such treatment proves to be inefficient, perilesional infiltrations of corticosteroids may be subscribed. These are only used systemically in those patients for whom all the previous courses of therapy have failed (7).

Its evolution varies according to the clinical type. Whereas reticular lesions cure spontaneously in $40 \%$ of the cases, atrophic and erosive lesions do not usually disappear and tend to recur in the majority of the cases (5). Some authors affirm that there is a $0.4 \%$ to $5 \%$ $(10,11)$ possibility of malignant transformation of oral lichen planus; therefore, performing periodic check-ups is of vital importance for these patients. 
The objective of this article is to highlight the most characteristic histopathological findings of oral lichen planus and their correlation with the most frequent clinical manifestations and forms in the sample.

\section{Material and Methods}

A retrospective study was carried out on a sample of 50 patients diagnosed with oral lichen planus. All of the patients were seen over a period of 11 years, from May 1998 to April 2009, at the University of Santiago de Compostela's Oral Medicine Teaching Unit of the School of Medicine and Dentistry. A complete clinical work-up was carried out on each patient based on an anamnesis (personal history, current illness, medication) and an intraoral and extraoral examination. The definitive diagnosis of lichen planus was obtained after completion of a biopsy and its subsequent anatomopathological study. Cases that did not meet both the clinical as well as the histological criteria specific to oral lichen planus were excluded from the analysis.

The clinical aspects of the disease (age, sex, clinical form and location of the lesions) and the histopathological findings observed at the epithelial level and in the connective tissue were then analyzed and compared. The statistical analysis of the data was performed using the Statistical Package for Social Sciences (SPSS), version 15.0. The results obtained are considered significant when $\mathrm{p}<0.05$.

\section{Results}

We analyzed a total of 50 patients, including 11 males $(22 \%)$ and 39 females $(78 \%)$. The age of the patients ranged from 28 to 82 years old, with an average age of 56.06 years old. The most frequent clinical type is reticular lichen planus, manifesting in 39 patients (78\%), followed by the erosive form in 7 cases (14\%) and the atrophic form in 4 cases $(8 \%)$. The most common loca- tion is in the posterior third of the buccal mucosa in $70 \%$ of the cases, which corresponds to 35 patients. We detected 8 cases $(16 \%)$ on the lingual dorsum, 3 cases $(6 \%)$ in the retrocomissural area, 2 cases $(4 \%)$ on the vestibular fundus, 1 case $(2 \%)$ in the gingiva and another case $(2 \%)$ located on the palate.

If we compare the clinical type of lichen planus with its location (Fig. 1), we note that the reticular form manifests more frequently in the posterior third of the buccal mucosa in $79.49 \%$ of the cases (31 patients), being located on the lingual dorsum in 5 patients $(12.82 \%)$, in the gingival area in 1 case $(2.56 \%)$, in the vestibular fundus in another patient $(2.56 \%)$, and in the retrocomissural area in 1 case $(2.56 \%)$. Similarly, erosive lichen planus is more common in the buccal mucosa, being identified in 4 patients $(57.14 \%)$, in the retrocomissural area in 2 cases $(28.57 \%)$, and on the palate in 1 patient $(14.29 \%)$. By contrast, the atrophic form is located more frequently on the lingual dorsum, as observed in 3 cases (75\%), and on the vestibular fundus in 1 patient $(25 \%)$.

Histologically, we analyzed the presence or absence of other anatopathological findings in the epithelium and connective tissue (Table 1). A hydropic degeneration of the basal layer of the epithelium was observed in all 50 patients (100\%). The presence of necrotic keratinocytes in the basal epithelial layer was identified in 46 patients $(92 \%), 36$ of which were in the reticular form $(78.26 \%)$, 6 erosive (13.04\%) and 4 atrophic $(8.69 \%)$. Therefore, $92.31 \%$ of the cases of reticular lichen planus, $85.71 \%$ of the erosive forms and $100 \%$ of the atrophic forms presented necrotic keratinocytes at the basal level.

No signs of epithelial dysplasia were observed in any of the samples; however, signs of atypia were noted at the basal layer in 2 cases of reticular lichen planus (4\%). Epithelial hyperplasia was identified in $54 \%$ of the patients (27 cases), 23 of which correspond to reticular lichen planus $(85.19 \%)$ and 4 of which correspond to the erosive form (14.81\%). We conclude that $59 \%$ of the re-

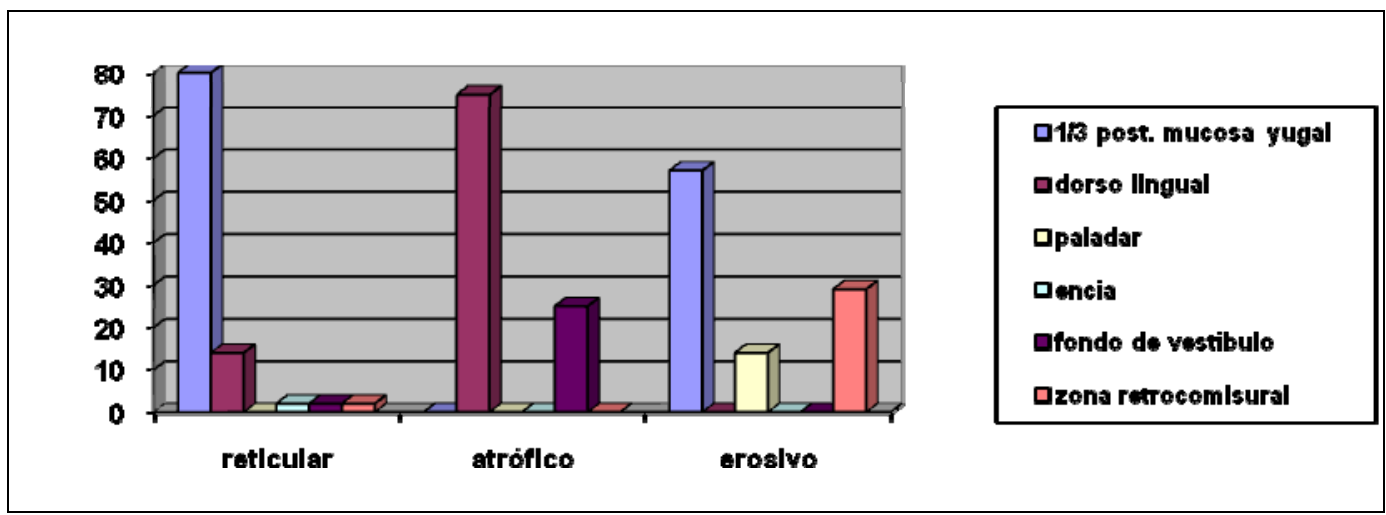

Fig. 1. Clinical form and location.

Reticular: reticular; atrófico: atrophic; erosivo: errosive; $1 / 3$ post. mucosa yugal: $1 / 3$ post. buccal mucosa; dorso lingual: lingual dorsum; paladar: palate; encía: gingiva; fondo de vestíbulo: vestibular fundus; zona retrocomisural: retrocomissural area. 
Table 1. Histological findings and clinical form of oral lichen planus.

\begin{tabular}{|l|c|c|c|}
\hline \multicolumn{1}{|c|}{ Histological findings/ Clinical form } & Reticular & Erosive & Atrophic \\
\hline Hydropic degeneration of the basal layer & $100 \%$ & $100 \%$ & $100 \%$ \\
\hline Necrotic keratinocytes in the epithelium & $92.31 \%$ & $85.71 \%$ & $100 \%$ \\
\hline Epithelial atypia & $5.12 \%$ & $0 \%$ & $0 \%$ \\
\hline Epithelial hyperplasia & $59 \%$ & $57.14 \%$ & $0 \%$ \\
\hline Epithelial hyperkeratosis & $69.23 \%$ & $57.14 \%$ & $50 \%$ \\
\hline Acanthosis & $48.71 \%$ & $57.14 \%$ & $25 \%$ \\
\hline Serrated ridges & $30.77 \%$ & $28.57 \%$ & $25 \%$ \\
\hline Fibrin deposit in the epithelium & $0 \%$ & $28.57 \%$ & $0 \%$ \\
\hline Epithelial erosion & $2.56 \%$ & $42.86 \%$ & $50 \%$ \\
\hline Neutrophils in the epithelium & $0 \%$ & $42.86 \%$ & $25 \%$ \\
\hline Subepithelial lymphocytic infiltrate & $100 \%$ & $100 \%$ & $100 \%$ \\
\hline Plasma cells in the connective tissue & $20.51 \%$ & $42.86 \%$ & $50 \%$ \\
\hline
\end{tabular}

ticular forms and $57.14 \%$ of the erosive forms presented hyperplasia at the epithelial level.

Hyperkeratosis was present in 33 of the samples $(66 \%)$, which included 27 cases of reticular lichen planus $(81.81 \%), 4$ erosive cases $(12.12 \%)$ and 2 atrophic cases $(6.06 \%)$. Thus, epithelial hyperkeratosis was present in $69.23 \%$ of the reticular forms, $57.14 \%$ of the erosive forms and $50 \%$ of the atrophic forms.

Acanthosis was identified in 24 cases (48\%); 19 corresponded to the reticular form $(79.16 \%), 4$ to the erosive form $(16.66 \%)$ and 1 to the atrophic form $(4.16 \%)$. Thus, acanthosis was present in $48.71 \%$ of the cases of reticular lichen planus, in $57.14 \%$ of the erosive cases and in $25 \%$ of the atrophic cases.

The presence of serrated ridges at the epithelial level was observed in 15 cases $(30 \%)$, which included 12 $(80 \%)$ cases of reticular lichen planus, $2(13.33 \%)$ cases of erosive lichen planus and 1 (6.66\%) case of atrophic lichen planus. Thus, saw tooth ridges were observed in $30.77 \%$ of the reticular form, in $28.57 \%$ of the erosive form and in $25 \%$ of the atrophic form of lichen planus. Fibrin deposits were identified at the epithelial level in 2 patients with erosive lichen planus (4\%).

Epithelial erosion was detected in 6 samples (12\%), 3 of which (50\%) involved the erosive form, $2(33.3 \%)$ involved the atrophic form and 1 sample $(16.66 \%)$ involved the reticular form. According to these figures, epithelial hyperkeratosis was present in $2.56 \%$ of the reticular forms, in $42.86 \%$ of the erosive forms and in $50 \%$ of the atrophic forms.

In 4 patients $(8 \%)$, the presence of neutrophils was observed at the epithelial level, 3 of which (75\%) corresponded to erosive lichen planus, and 1 (25\%) corresponding to the atrophic form.

In the subepithelial connective tissue, a band-like lymphocyte infiltrate $\mathrm{T}$ was observed in $100 \%$ of the samples. This lymphocytic infiltrate extends toward the basal layer of the epithelium in 22 patients (44\%) and in depth with perivascular distribution in 1 case $(2 \%)$.

The presence of plasma cells in the connective tissue was detected in 13 patients (26\%); of these, $8(61.53 \%)$ had reticular lichen planus, 3 (23.07\%) had erosive lichen planus and $2(15.38 \%)$ had atrophic lichen planus. Thus, these cells were observed in $20.51 \%$ of the reticular forms, in $42.86 \%$ of the erosive forms and in $50 \%$ of the atrophic forms.

If we compare the most common clinical form and location with the histological findings (Fig. 2), we observe

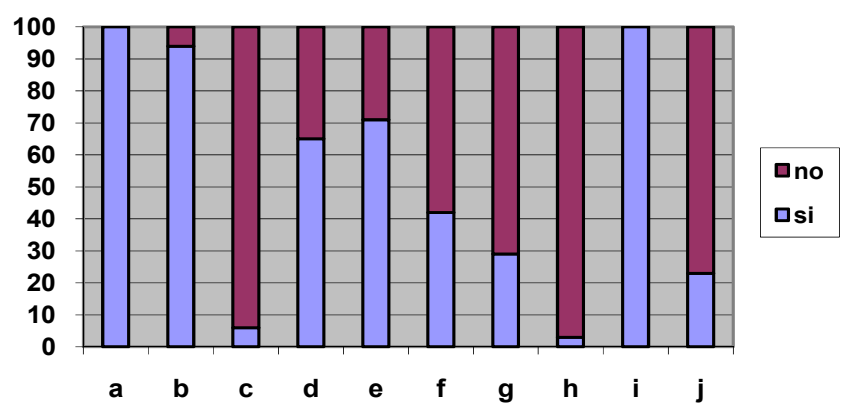

a: hydropic basal degeneration

b: necrotic keratinocytes

c: epithelial atypia

d: epithelial hyperplasia

e: epithelial hyperkeratosis

f: acanthosis

g: serrated ridges

$\mathbf{h}$ : epithelial erosion

i: lymphocytic infiltration in the subepithelial

layer

j: plasma cells

Sí: Yes; No: No

Fig. 2. Histological findings in reticular oral lichen planus. 
that in reticular lichen planus located in the posterior third of the mouth, hydropic degeneration of the basal layer of the epithelium and band-like subepithelial lymphocytic infiltration is identified in $100 \%$ of the cases; necrotic keratinocytes at the epithelial level are observed in $93.5 \%$ of the patients; epithelial hyperkeratosis is present in $71 \%$; epithelial hyperplasia in $64.5 \%$; acanthosis in $41.9 \%$; serrated ridges in the $29 \%$ of the patients; presence of plasma cells in $22.6 \%$; atypical squamous cells without dysplasia is present in $6.4 \%$ of the patients and epithelial erosion is observed in $3.2 \%$ of the cases.

\section{Discussion}

The importance of oral lichen planus is proportional to its degree of frequency and incidence, its similarity with other diseases of the mucosa, its occasionally painful nature and its possible connection with malignant tumors.

We agree with other authors (3) that lichen planus occurs more frequently in females and manifests between $50-60$ years of age. In fact, in our study, $78 \%$ of the patients are females and the average age for both sexes is 56.06 years old. However, the disease can develop at any age, such that the age range of our sample varies from 28 to 82 years old, which is consistent with other studies (12).

We agree with other authors $(1,7)$ that the most frequent clinical form is reticular lichen planus. In our study, $78 \%$ of the cases correspond to reticular lichen planus, whereas the erosive form is observed in $14 \%$ of the patients and the atrophic form in $8 \%$ of the patients, respectively.

As has been shown in other studies (3), the most common location of the lesions is in the buccal mucosa, with the next most common location being the tongue. In our analysis, the buccal mucosa was the most common location in $70 \%$ of the cases, followed by the tongue in $16 \%$ of the cases-the other locations being less common.

Another fact that we have observed is the predisposition of its location according to the clinical type. In this study, which concurs with that of several authors (1), we observed that the most common location of the reticular form is in the buccal mucosa, with percentages of $79.49 \%$, just as with the erosive type in $57.14 \%$ of the cases. By contrast, atrophic lichen planus was located on the tongue in $75 \%$ of the patients.

Focusing on the histological findings, hydropic degeneration of the basal layer of the epithelium and the bandlike subepithelial lymphocytic inflammatory infiltrate $\mathrm{T}$ is identified in $100 \%$ of the patients, a finding that is corroborated by other authors (9) and which, along with the absence of epithelial dysplasia, constitutes the three typical histological criteria of oral lichen planus.

In $4 \%$ of the cases, we observed signs of epithelial aty- pia in the form of enlarged pyknotic nuclei, but no signs of dysplasia were detected at this level. The presence of necrotic keratinocytes has been described by various authors (13) as a result of the hydropic degeneration of the basal cells. In our study, these necrotic cells were identified in $92 \%$ of the patients.

Epithelial hyperplasia is a finding that we observed in $54 \%$ of the sample. However, it was not identified in any of the atrophic forms. We were unable to compare this particular fact with any author; however, it is true that Bagan et al. (14) showed that the thickness and length of the epithelium is greater in the reticular forms than in that of the atrophic and erosive forms.

Epithelial hyperkeratosis was observed in $66 \%$ of our patients, being more predominant in the reticular form. This increased thickness of the corneal layer has also been described in several other studies $(15,16)$.

As regards acanthosis, our data is consistent with that found in the literature $(15,16)$, with percentages of approximately $48 \%$.

The presence of serrated ridges is another one of the histological findings of oral lichen planus observed in $30 \%$ of our sample and described by other authors (15). Fibrin deposits in the epithelium are a phenomenon that we found in $4 \%$ of our cases, all of which corresponded to the erosive form. We found data in the literature with regard to this substance, identifying fibrin in the perilesional mucosa using immunofluorescence techniques (17).

Epithelial erosion is a finding that is relatively more common in the atrophic and erosive forms, observed in only $2.56 \%$ of the cases of reticular lichen planus. This could be because, as some authors claim (14), the thickness of the epithelium is greater in the reticular forms, with a thinning observed in the atrophic and erosive forms, therefore making them more prone to erosion.

Neutrophils were identified at the epithelial level in $8 \%$ of our patients. In all of the cases, it corresponds with the atrophic and erosive forms-no cases being observed in the reticular form. The presence of eosinophilic cells has been previously reported in the literature by authors such as Hall et al. (16).

As for the subepithelial connective tissue, we observed the presence of band-like $\mathrm{T}$ lymphocytes and plasma cells, a finding that is consistent with previous studies (16). In $44 \%$ of the cases, there was an extension of the inflammatory infiltrate into the basal layer of the epithelium; and in $2 \%$ of the patients, the extension was in depth and with perivascular distribution. This phenomenon may be because, as stated by Eisenberg (18), in lichen planus lesions there is a separation between the epithelium and the lamina itself, which facilitates the penetration of subepithelial inflammatory infiltrate cells.

In conclusion, oral lichen planus is a disease that is more 
common among females, usually occurring in the 5th and 6 th decades of life. The most common clinical form is reticular lichen planus, primarily manifesting in the buccal mucosa. The histological findings characteristic of oral lichen planus are hydropic degeneration of the basal layer, a band-like subepithelial lymphocyte infiltration and the absence of epithelial dysplasia. However, at the epithelial level, it is also common to observe phenomena such as hyperplasia, hyperkeratosis, acanthosis and the presence of necrotic keratinocytes.

\section{References}

References with links to Crossref - DOI

1. Ismail SB, Kumar SK, Zain RB. Oral lichen planus and lichenoid reactions: etiopathogenesis, diagnosis, management and malignant transformation. J Oral Sci. 2007:49:89-106.

2. Scully C, Beyli M, Ferreiro MC, Ficarra G, Gill Y, Griffiths M, et al. Update on oral lichen planus: etiopathogenesis and management. Crit Rev Oral Biol Med. 1998;9:86-122.

3. Bagán-Sebastián JV, Milián-Masanet MA, Peñarrocha-Diago M, Jiménez Y. A clinical study of 205 patients with oral lichen planus. J Oral Maxillofac Surg. 1992;50:116-8.

4. Porter SR, Kirby A, Olsen I, Barrett W. Immunologic aspects of dermal and oral lichen planus: a review. Oral Surg Oral Med Oral Pathol Oral Radiol Endod. 1997;83:358-66.

5. Andreasen JO. Oral lichen planus. 1. A clinical evaluation of 115 cases. Oral Surg Oral Med Oral Pathol. 1968;25:31-42.

6. Silverman S Jr, Gorsky M, Lozada-Nur F. A prospective followup study of 570 patients with oral lichen planus: persistence, remission, and malignant association. Oral Surg Oral Med Oral Pathol. 1985;60:30-4.

7. Eisen D, Carrozzo M, Bagan Sebastian JV, Thongprasom K. Number V Oral lichen planus: clinical features and management. Oral Dis. 2005;11:338-49.

8. Al-Hashimi I, Schifter M, Lockhart PB, Wray D, Brennan M, Migliorati CA, et al. Oral lichen planus and oral lichenoid lesions: diagnostic and therapeutic considerations. Oral Surg Oral Med Oral Pathol Oral Radiol Endod. 2007;103 Suppl:S25.e1-12.

9. Van der Meij EH, Van der Waal I. Lack of clinicopathologic correlation in the diagnosis of oral lichen planus based on the presently available diagnostic criteria and suggestions for modifications. J Oral Pathol Med. 2003;32:507-12.

10. Van der Meij EH, Schepman KP, Smeele LE, Van der Wal JE, Bezemer PD, Van der Waal I. A review of the recent literature regarding malignant transformation of oral lichen planus. Oral Surg Oral Med Oral Pathol Oral Radiol Endod. 1999;88:307-10.

11. Pakfetrat A, Javadzadeh-Bolouri A, Basir-Shabestari S, Falaki F. Oral Lichen Planus: a retrospective study of 420 Iranian patients. Med Oral Patol Oral Cir Bucal. 2009;14:E315-8.

12. Scully C, de Almeida OP, Welbury R. Oral lichen planus in childhood. Br J Dermatol. 1994;130:131-3.

13. Shklar G. Lichen planus as an oral ulcerative disease. Oral Surg Oral Med Oral Pathol. 1972;33:376-88.

14. Bagan Sebastian JV, Aguirre Urizar JM, Milián Masanet A, Peñarrocha Diago M, García Pola Vallejo MJ. A morphometric study of 74 cases of oral lichen planus. Rev Stomatol Chir Maxillofac. 1991;92:265-8.

15. Batra P, Wang N, Kamino H, Possick P. Linear lichen planus. Dermatol Online J. 2008;14:16.

16. Hall R, Wartman D, Jellinek N, Robinson-Bostom L, Telang G. Lichen planus of the nail matrix with predominant plasma cell infiltrate. J Cutan Pathol. 2008;35 Suppl 1:14-6.

17. Helander SD, Rogers RS 3rd. The sensitivity and specificity of direct immunofluorescence testing in disorders of mucous membranes. J Am Acad Dermatol. 1994;30:65-75.

18. Eisenberg E. Oral lichen planus: a benign lesion. J Oral Maxillofac Surg. 2000;58:1278-85

\section{Acknowledgments}

With the support of the European Social Fund and the "Dirección Xeral de Ordenación e Calidade do Sistema Universitario de Galicia" of the Department of Education and Universities - Xunta de Galicia (Autonomous Government of Galicia) 